\title{
Evaluation of Age Related Changes Associated with Exposure of Wistar Rats to Dichlorvor on some Haematological Parameters
}

\author{
${ }^{* 1}$ IGE, SF; ${ }^{1}$ SERIKI, MA; ${ }^{2}$ OLATEJU, BS; ${ }^{1}$ OLADIPUPO, VA \\ ${ }^{I}$ Department of Physiology, Faculty of Basic Medical Sciences, College of Health Sciences, \\ Ladoke Akintola University of Technology, P.M.B. 4000, Ogbomoso, Oyo State, Nigeria. \\ ${ }^{2}$ Department of Physiology, College of Medicine University of Lagos, Lagos State, Nigeria. \\ *Corresponding Author Email: sfige@lautech.edu.ng; funkeige2006@yahoo.com,Tel: +2348060743616
}

\begin{abstract}
Different studies proved that exposure over prolonged periods to pesticides increase the likelihood of developing adverse effects. 2,2-dichlorovinyl dimethyl phosphate (DDVP) is a predominant pesticide used in controlling insects. Therefore this study was aimed to investigate the age related changes in hematological indices of animals exposed to DDVP. Thirty healthy male Wistar rats were used in this study. Animals were grouped into three major groups of ten animals each. Group one animals served as young group (5 - 6 weeks) while group two and three was the middle-aged (2 months) and old groups (3-4 months) respectively. All the groups were subdivided into control and DDVP group with five animals in each subgroup. The DDVP groups were exposed to DDVP for five weeks. At the end of fifth week, they were euthanized with blood collected through cardiac puncture, hematological indices were analysed with auto hematological analyzer.Exposure to DDVP significantly increased white blood cell counts and basophiles in old group. Platelets count and plateletcrit (PCT) were significantly decreased in young animals that were exposed to DDVP while platelet distribution width (PDW) and Mean platelets volume (MPV) were not significantly different across the groups. Red blood cells count, Hematocrit, Hemoglobin concentration, Mean cell volume, Mean cell hemoglobin and Mean cell hemoglobin concentration also show no significant difference across groups. Exposure of Wistar rats to Dichlorvos has no impact on haematological parameters in middle agerats. However, dichlorvos affects young and old animals proving age dependent effect of DDVP on hematological parameters.
\end{abstract}

\section{DOI: https://dx.doi.org/10.4314/jasem.v25i3.27}

Copyright: Copyright $(92021$ Ige et al. This is an open access article distributed under the Creative Commons Attribution License (CCL), which permits unrestricted use, distribution, and reproduction in any medium, provided the original work is properly cited.

Dates: Received: 12 December 2020; Revised: 26 January 2021; Accepted: 12 February 2021

Keywords: DDVP, hematology, white blood cells, platelet, age

2, 2-dichlorovinyl dimethyl phosphate otherwise known as dichlorvos is a predominant insecticide used in controlling insects particularly in developing countries, (Okoroiwu and Iwara, 2018). It is an organophosphate insecticide that is traded under names such as Sniper, Nogos, Dedevap, DDVP, Phosvit, Nuval, and Daksh, (Owoeye et al., 2014). In 1992, WHO classified DDVP as a "highly hazardous" chemical, (WHO, 1995). Since 1961, DDVP has been in commercial production and is used in households as pesticides, outdoor areas, and in aircraft, (Edem et al., 2012). Also, it is used as agricultural chemical for the control of pests. The routes of absorption of DDVP are inhalation, ingestion, or absorption through the skin, (Okoroiwu and Iwara, 2018). Among the organophosphates, DDVP shows rapid metabolism and excretion in mammals and even after rats and mice were exposed to 17 times the quantity used for insect control in homes, dichlorvos was not detected in their blood. The reason for this is because of degrading enzymes present in blood plasma and tissues, (Hayes and Laws, 1990). Even though liver is the major site for its detoxification, spleen, lung, blood, and kidney also metabolize it, (Gains et al., 1996). There are different studies that proved that exposure over prolonged periods to pesticides increases the likelihood of developing adverse immunological, developmental, and reproductive effects as well as neurotoxicity,( Zahm and Ward, 1998; Jenner, 2001). Other effects include prostate cancer, brain cancer, and leukemia, (OjajarviI et al., 2000; Mourad, 2005). Dichlorvos shows toxicity by blocking the enzyme responsible for the degradation of acetylcholine, acetylcholinesterase (Edem et al., 2012; Imam et al., 2018) thereby leading to the excessive amount of acetylcholine in muscular motor plates, nervous tissue with symptoms of endogenic poisoning(Edem et al., 2012).Few studies have been carried out on the effect of pesticides on haematological parameters. Prolonged exposure to pesticides among the farmers has been linked to thrombocytopenia, neutropenia, agranulocytosis, aplastic anaemia, multiple myeloma and chronic lymphoid leukemia (WHO, 1995; Joshaghani et al., 2007; Piccoli et al., 2019) $)^{1}$ Brown et 
al. (2015) had also found a significant decrease in mean values of hemoglobin, packed cell volume, and red blood cells in albino rats that were exposed to dichlorvos. Different studies that evaluated the effects of age on absorption, distribution, metabolism, and elimination of chemicals only concentrated on drugs instead of toxic environmental substances. It is known that these toxicokinetic measures are able to change with age (Mortuza et al.,2018). Furthermore, toxicity is mostly measured in young animals and therefore, it is necessary to know response in neonates and old age. Despite the wide use of DDVP, there is limited knowledge about the haematological indices of people exposed to the chemical, (Edem et al., 2012). Furthermore, how age influence the effect of DDVP on blood characteristics has not yet been clearly studied. Such information is highly needed so as to help formulate policy that will protect different age groups from effect of exposure to pesticides. Therefore, the objective of this paper is to evaluate the age related changes associated with the effect of 2,2dichlorovinyl dimethyl phosphate on some haematological parameters of Wistar rats exposed to Dichlorvors.

\section{MATERIALS AND METHODS}

Animals: Thirty healthy male Wistar rats were used in this study. They were kept in polyvinyl wire mesh cages in the animal house of Department of Physiology, Ladoke Akintola University of Technology, Ogbomoso, Nigeria. They have free access to food and water under standard laboratory conditions of $12 \mathrm{~h}$ light and dark cycle. Animals were grouped into three major groups of ten animals each. Group one animals served as young rats group while group two and three was the middle-aged and old groups respectively. All the groups were subdivided into control and DDVP group with five animals in each subgroup. Animals were 5 - 6 weeks, 2 months and 3 4 months old in young and middle-aged and old group respectively (Pallav, 2013) at the beginning of this study. Animals' weights were measured before exposure and at the end of the three and five weeks of experiment.

Dichlorvos exposure: Rats in DDVP groups were exposed to DDVP from 8a.m to 12noon for five weeks. Dichlorvos was purchased from agrochemical shop in Ogbomosho, Oyo State, Nigeria. Preparation and exposure of the animals to dichlorvos was according to method reported by Edem et al., 2012. On each day of exposure, experimental groups in their respective cages were placed in a poorly ventilated compartment. Dichlorvos solution was prepared by mixing $50 \mathrm{ml}$ of dichlorvos with $50 \mathrm{ml}$ of distilled water. On each day of exposure, dichlorvos solution was freshly prepared.
Sample collection and Haematological Analysis: Blood was collected by cardiac puncture into EDTA bottles. Blood collected was analyzed by Mindray auto haematological analyzer (Shenzhen Mindray BioMedical electronics, Co., LTD, China).

Statistical Analyses: Data were expressed as mean \pm standard error of mean (Mean \pm S.E.M). Analyses of data were done with Graph Pad Prism version 5 software. Significant difference among the groups were compared by one-way analysis of variance (ANOVA) using Tukey's multiple comparison test as post hoc test. Statistical significance was set at $\mathrm{p}<0.05$.

\section{RESULTS AND DISCUSSION}

Weight gain of animals exposed to DDVP: There was no significant difference in weight gain of animals exposed to DDVP and control in young and middleaged groups but there was significant reduction in weight gain of old animal that was exposed to DDVP after three weeks. Significant reduction was also found in animals that were exposed to DDVP for five weeks in young and old animals groups when compared them with their respective control, Figure 1 and 2.

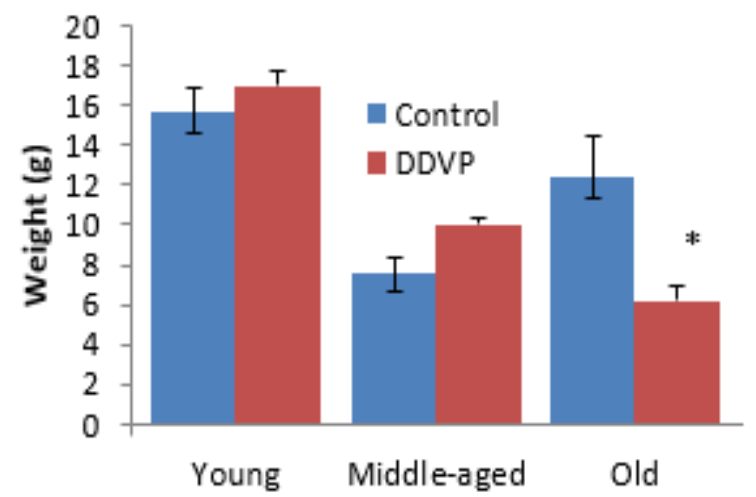

Fig 1: Weight gain after three weeks of exposure to DDVP $(* \mathrm{p}<$ 0.05 vs control)

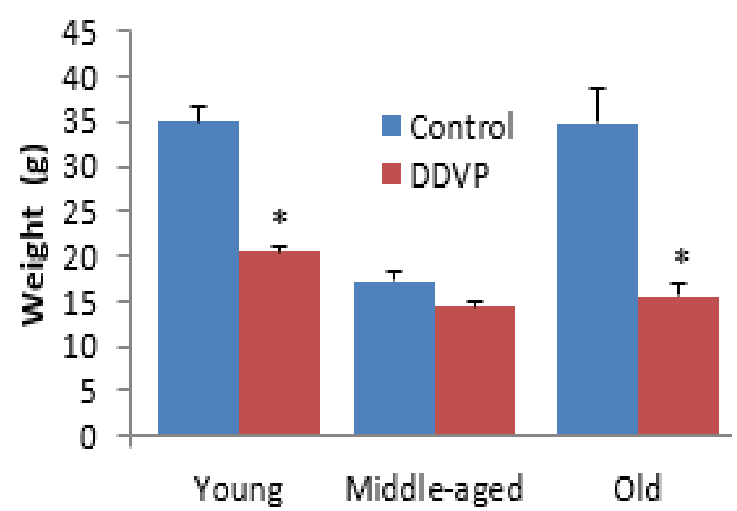

Fig 2: Weight gain after five weeks of exposure to DDVP $\left({ }^{*} \mathrm{p}<\right.$ 0.05 vs control) 
Effect of DDVP on WBC and differential count: White blood cells (WBC) counts were significantly increased in old animals as a result of exposure to DDVP, while there was no significant change in while blood cells count of young and middle-aged exposed to DDVP when compared with their control, Figure 3 . The differential white blood cell count revealed significant increase in basophiles of old animals while there was no significant different in neutrophils, lymphocyte, monocyte and eosinophils in young and middle-aged exposed to DDVP when compared with their control. There was no significant change in basophils of young and middle-aged exposed to DDVP and their control, Table 1 .

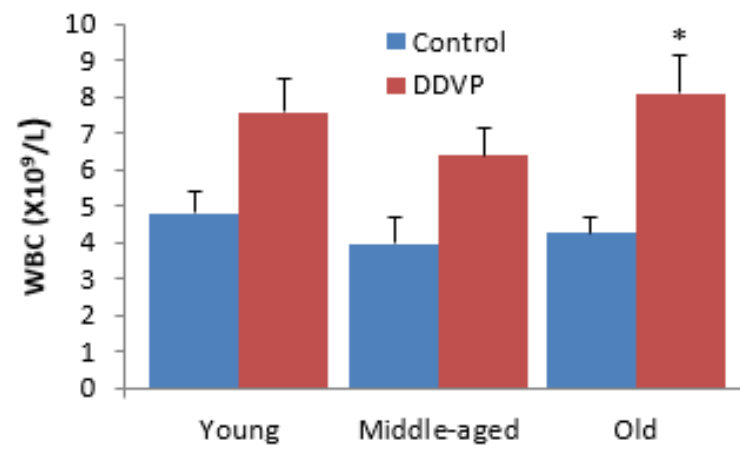

Fig 3: Effect of DDVP on white blood cell count of different age groups

Red blood indices of animals exposed to DDVP: Red blood cells count (RBC), Hematocrit (HCT), Hemoglobin concentration (HGB), Mean cell volume $(\mathrm{MCV})$, Mean cell hemoglobin $(\mathrm{MCH})$, Mean cell hemoglobin concentration (MCHC) and Red cell distribution - co efficient variant (RDW-CV )were not significantly different across the groups. However, the red cell distribution standard deviation (RDW-SD) of the old animals was significantly increased when compared with their control, young and middle-aged animals' red cell distribution standard deviation that were exposed to DDVP. These details are found in Table 2.

Effect of DDVP on platelets indices: Platelets count and plateletcrit (PCT) were significantly decreased in young animals that were exposed to DDVP when compared with their controls. Platelet distribution width (PDW) and Mean platelets volume (MPV) were not significantly different across the groups. In the present study old animals that were exposed to DDVP showed significant reduction in weight gain as compared to their control groups. This weight reduction was observed from week three of exposure. In young rats, there was significant reduction in weight gain at week five showing that at acute exposure, the young animals were able to cushion the effect of DDVP on their weights, Figure 1 and 2. Erythrocytes indices: RBC, HGB, HCT, MCV, $\mathrm{MCH}$, and $\mathrm{MCHC}$ are not altered by exposure to DDVP in the present study. This finding is in agreement with work of Celik et al (2009), where animals were exposed to 5 and 10 ppm of dichlorvos by ingestion. However, Edem et al. (2012) had reported a significant reduction in HCT and $\mathrm{MCV}$ and significant increase in MCHC of animals exposed to DDVP. These details are found in table 1 and figure 5.Intact hemoglobin concentration reported in this study shows that iron metabolism is not altered. 3.

Table 1: Effect of DDVP on differential leucocyte count of different age groups

\begin{tabular}{llllll}
\hline Group/Parameters & Neutrophils & Lymphocytes & Monocytes & Eosinophils & Basophils \\
\hline Young control & $22.33 \pm 2.88$ & $71.1 \pm 4.41$ & $6.78 \pm 4.51$ & $0.48 \pm 0.21$ & $1.1 \pm 0.20$ \\
Young DDVP & $20.63 \pm 0.60$ & $79.06 \pm 1.93$ & $3.88 \pm 2.21$ & $0.32 \pm 0.13$ & $1.24 \pm 0.19$ \\
Middle - age control & $27.63 \pm 3.10$ & $72.44 \pm 2.79$ & $4.70 \pm 1.99$ & $0.30 \pm 0.07$ & $1.50 \pm 0.26$ \\
Middle - age DDVP & $19.63 \pm 1.04$ & $78.08 \pm 0.76$ & $2.92 \pm 1.97$ & $0.28 \pm 0.13$ & $1.70 \pm 0.29$ \\
Old control & $13.45 \pm 2.34$ & $72.42 \pm 2.05$ & $8.48 \pm 3.27$ & $0.50 \pm 0.12$ & $1.34 \pm 0.24$ \\
Old DDVP & $12.63 \pm 4.16$ & $76.22 \pm 5.71$ & $2.98 \pm 1.10$ & $0.36 \pm 0.14$ & $2.54 \pm 0.22^{*}$ \\
\hline
\end{tabular}

Table 2: Effect of DDVP on erythrocyte indices of different age groups

\begin{tabular}{lllllllll}
\hline $\begin{array}{l}\text { Group/Para } \\
\text { meters }\end{array}$ & $\begin{array}{l}\text { RBC } \\
\left(\mathrm{X} 10^{12}\right)\end{array}$ & $\begin{array}{l}\mathrm{HGB} \\
(\mathrm{g} / \mathrm{dL})\end{array}$ & $\begin{array}{l}\mathrm{HCT} \\
(\%)\end{array}$ & $\begin{array}{l}\mathrm{MCV} \\
(\mathrm{fL})\end{array}$ & $\begin{array}{l}\mathrm{MCH} \\
(\mathrm{pg})\end{array}$ & $\begin{array}{l}\text { MCHC } \\
(\mathrm{g} / \mathrm{dL})\end{array}$ & $\begin{array}{l}\text { RDW- } \\
\mathrm{CV}(\%)\end{array}$ & $\begin{array}{l}\text { RDW- } \\
\text { SD }(\mathrm{fL})\end{array}$ \\
\hline Young & $6.73 \pm$ & $13.70 \pm$ & $45.42 \pm$ & $67.54 \pm$ & $20.36 \pm$ & $30.18 \pm$ & $13.7 \pm 0$. & $39.72 \pm$ \\
control & 0.31 & 0.56 & 2.11 & 1.16 & 0.21 & 0.37 & 23 & 1.78 \\
Young & $7.26 \pm$ & $14.26 \pm$ & $47.62 \pm$ & $65.60 \pm$ & $19.66 \pm$ & $29.98 \pm$ & $13.85 \pm$ & $39.98 \pm$ \\
DDVP & 0.21 & 0.17 & 0.95 & 0.69 & 0.38 & 0.38 & 0.30 & 0.60 \\
Middle - & $6.28 \pm$ & $13.36 \pm$ & $43.28 \pm$ & $66.88 \pm$ & $22.06 \pm$ & $30.96 \pm$ & $14.18 \pm$ & $40.32 \pm$ \\
age control & 0.50 & 0.40 & 1.7840. & 8.08 & 2.59 & 0.70 & 0.40 & 1.50 \\
Middle - & $6.15 \pm$ & $12.40 \pm$ & $44.92 \pm$ & $64.46 \pm$ & $20.10 \pm$ & $31.34 \pm$ & $13.45 \pm$ & $39.74 \pm$ \\
age DDVP & 0.86 & 1.76 & 1. & 1.21 & 0.15 & 0.42 & 0.24 & 1.72 \\
Old control & $6.88 \pm$ & $14.04 \pm$ & $46.48 \pm$ & $68.50 \pm$ & $21.62 \pm$ & $31.34 \pm$ & $14.90 \pm$ & $39.63 \pm$ \\
& 0.73 & 0.28 & 1. & 7.86 & 2.89 & 0.68 & 0.28 & 1.23 \\
Old DDVP & $7.01 \pm$ & $13.63 \pm$ & 68 & $66.34 \pm$ & $19.58 \pm$ & $29.48 \pm$ & $15.7 \pm 0$. & $47.24 \pm$ \\
& 0.21 & 0.43 & \multicolumn{7}{c}{$* 1.83$} & 0.72 & 0.48 & 24 & $1.03 *$ \\
\hline
\end{tabular}

IGE, SF; SERIKI, MA; OLATEJU, BS; OLADIPUPO, VA 
Table 3: Effect of DDVP on platelets count, mean platelets volume, PDW and PCT of different age groups

\begin{tabular}{lllll}
\hline Group/Parameters & PLT $\left(\mathrm{x} 10^{9} / \mathrm{L}\right)$ & $\mathrm{MPV}(\mathrm{fL})$ & $\mathrm{PDW}$ & $\mathrm{PCT}(\%)$ \\
\hline Young control & $704.25 \pm 39.43$ & $7.72 \pm 0.08$ & $19.92 \pm 0.21$ & $0.57 \pm 0.04$ \\
Young DDVP & $511.00 \pm 8.16^{*}$ & $8.30 \pm 0.11$ & $15.74 \pm 0.02$ & $0.39 \pm 0.01^{*}$ \\
Middle - age control & $560.33 \pm 37.32$ & $7.86 \pm 0.35$ & $15.94 \pm 0.19$ & $0.45 \pm 0.04$ \\
Middle - age DDVP & $511.00 \pm 1057$ & $8.24 \pm 0.09$ & $15.60 \pm 0.05$ & $0.40 \pm 0.02$ \\
Old control & $703.33 \pm 17.36$ & $8.15 \pm 0.35$ & $15.48 \pm 0.04$ & $0.52 \pm 0.03$ \\
Old DDVP & $538.67 \pm 19.10$ & $8.44 \pm 0.18$ & $15.84 \pm 0.22$ & $0.50 \pm 0.04$ \\
\hline \multicolumn{5}{c}{$* \mathrm{P}<0.05$ vs control }
\end{tabular}

A further study on how DDVP affects factors necessary for hemoglobin synthesis may help explain reason for the difference between the findings of this study and Edem et al. (2012). White blood cells were significantly increased in old animals as a result of exposure to DDVP. There was no significant change in while blood cells count of young and middle-aged rats exposed to DDVP when compared to their respective control. This shows that DDVP triggers immune response more in old animals compared to young and middle-aged rats. In a similar study, Edemet al. (2012)did not find significant difference in leukocytes indices of adult animals who were exposed to DDVP and those that are not exposed. The study of Edemet al. (2012) did not however consider the impact of DDVP in old animals. Increased immune response in old animals may be as a result of increase retention of the toxin due to impaired ability of the liver to metabolize and kidney to excrete the toxin, (Kanuet al. 2016). Furthermore, old age makes animals to be more prone to infection. The increased leukocytes may therefore be to protect the body against infections caused by dichlorvos. The differential white blood cell count also revealed significant increase in basophiles of old animals while there was no significant different in neutrophils, lymphocyte, monocyte and eosinophils in young, middle-aged and old animals exposed to DDVP when compared with their control, Figure 3 and 4. The increased in basophils may be caused by overproduction of white blood cells found in old animals. Table 3 shows that platelets count and plateletcrit (PCT) were only significantly decreased in young animals that were exposed to DDVP while platelet distribution width (PDW) and Mean platelets volume (MPV) were not significantly different across the groups. Our result on platelet indices is in tandem with the study of Varolet al. (2012), they have found platelet indices to be significantly lower in farm workers exposed to pesticides.

Conclusion: Exposure of Wistar rats to Dichlorvosfor five weeks have no impact on haematological parameters of middle age rats. However, dichlorvos affects young (platelet indices) and old animals proving that age is an important factor to be considered in the selection of animals in any study that involves pesticides. There is a need for further research that measures impact of Dichlorvos on factors affecting these haematological parameters.

\section{Acknowledgments}

We sincerely acknowledge MrAjetunmobiof

LAUTECH teaching hospital Ogbomoso, Oyo State,

Nigeria for his technical support.

\section{REFERENCES}

Brown, H; Kenanagha, B and Onwuli, DO (2015). Haemato-pathological effect of dichlorvos on blood picture and liver cells of albino rats.J.Toxicol.Environ. Health Sci.7(2): 18-23.

Celik, I;Yilmaz, Z; Turkoglu, V (2009).Hematotoxic and hepatotoxic effects of dichlorvos at sublethal dosages in rats. Environ Toxicol 24: 128-132

Edem, VF; Akinyoola, SB; Olaniyi, JA; Rahamon, SK; Owoeye, O; Arinola, OG (2012).Haematological Parameters of Wistar Rats Exposed to 2, 2 Dichlorovinyl Dimethyl Phosphate Chemical. Asian J. Exp. Biol. Sci 3(4): 838 - 841

Gains, TB; Hayes, WJ; Linder, RE (1996). Liver metabolism of anticholinesterase compounds in live rats: In relation to toxicity. London: Nature; p. 2098889.

Hayes, WJ; Laws, ER (1990).Handbook of pesticide toxicology. New York: Academic press Inc; vol. 3

Imam, A; Busari, MO; Adana, MY; Ajibola, MI; Ibrahim, A; Suleimon, FA; Ajao, MS (2018). Subchronicdichlorvos-induced Cardiotoxicity in Wistar rats: Mitigative efficacy of Nigella sativa oil. J. Exp.Clin.Anat 17:60-5

Jenner, P (2001). Parkinson's disease, pesticides and mitochondria ldysfunction. Trends in Neurosciences24:245-247.

Joshaghani, HR; Ahmadi, AR;Mansourian, MR (2007). Effects of occupational exposure in pesticide plant on workers serum and erythrocyte 
cholinesterase activity. Int. J.Occup. Med. Environ. Health. 20(4):381-5

Kanu, K; Ijioma, S;Atiata, O (2016).Haematological, Biochemical, and Antioxidant changes in Wistar Rats exposed to Dichlorvos Based Insecticide Formulation in Southeast Nigeria. Toxics 4(4): 28

Mortuza, T; Chen, C; White, C; Cummings, B; Bruckner, J (2018).Toxicokinetics of Deltamethrin; Dosage Dependency. Vehicle Effects, and Low-Dose Age-Equivalent 336 Dosimetry in Rats. Toxicol Sci.162(1): 327 -

Mourad, LA. (2005). Adverse impact of insecticides on the health of Palestian farm workers in the Gaza Strip: A hematologic biomarker study. International Journal of Occup Environ Med.11:144-9.

Ojajarvi, I; Partamen, T; Ahilbom, A; Boffetta, P; Hakulimen, T;Jourenkova, N (2000). Occupational exposures and pancreatic cancer: A meta analysis. Occup Environ Med. 7:316-324

Okoroiwu, HU and Iwara, IA (2018).Dichlorvos toxicity: A public health perspective. Interdiscip. Toxic. 11(2), 129-137
Owoeye, O; Edem, F; Akinyoola, B;Arinola, G (2014). Renal Corpuscles Were Protected From Dichlorvos-Induced Morphological Alterations in Rats by Antioxidant Vitamins. Int. J. Morphol 32(2): 475-480

Pallav, S (2013). The Laboratory Rat: Relating Its Age with Humans. Int J Prev Med 4(6): 624-630.

Piccoli, C; Cremonese, C; Koifman, R; Koifman, S; Freire, C (2019). Occupational exposure to pesticides and haematological alterations: A survey of farm residents in the South of Brazil.Ciênc. saúdecoletiva 24(6), 2325-2340.

Varol,E;Ogut, S;Gultekin, F(2014). Effect of pesticide exposure on platelet indices in farm workers. Toxic. Ind. Health 30 (7):630-4.

World Health Organization (1995). International Programme on chemical safety. WHO recommended classification of pesticide by hazards and guidelines to classification 19941995.UNEP/ILO/WHO.

Zahm, SH and Ward, MH (1998). Pesticides and childhood cancer. Environ Health Perspect106:893-908. 